\title{
Вопросы оценки видимости с учетом влияния увлажненного аэрозоля и температуры воздуха
}

\author{
Ф.Г. Абасзаде, Х. Г. Асадов \\ Национальное аэрокосмическое агентство, Республика Азербайджан \\ (AZ1115, г. Баку, ул. С. С. Ахундова, 1)
}

\begin{abstract}
Аннотация: Статья посвящена оценке видимости с учетом влияния увлажненного аэрозоля и температуры воздуха. Хорошо известно, что видимость зависит от оптических свойств компонентов атмосферы, физических свойств наблюдаемого объекта, а также порогового визуального контраста наблюдателя. Целью настоящей статьи является изучение совместной зависимости метеорологической видимости как от относительной влажности, так и от температуры воздуха. Рассмотрен сценарий метеорологических измерений когда осуществляется много шаговые измерения показателя относительной влажности $R H\left(T_{x}\right)$ в интервале температур $\left(T_{x}-T_{0}\right)$ с равным шагом $\Delta T$. Показано, что для достижения максимальной информативности проводимых измерений температуру $T_{x}$ следует выбирать таким образом, чтобы $R H\left(T_{0}\right)$ был пропорционально значению $\mathrm{C}_{1}\left(T_{x}-T_{0}\right)$. Таким образом, определен порядок проведения многошаговых измерений видимости в условиях увеличения температуры воздуха во времени. Согласно вычисленной закономерности при каждом шаге измерения вновь введенного показателя, являющеюся смещенной величиной относительной видимости, величина приращения температуры в следующем шаге должна быть выбрана пропорционально величине относительной влажности в текущем шаге измерений.
\end{abstract}

Ключевые слова: видимость, относительная влажность, оптимизация, аэрозоль, увлажнение, измерения.

Для цитирования: Абасзаде Ф.Г., Асадов Х.Г. Вопросы оценки видимости с учетом влияния увлажненного аэрозоля и температуры воздуха // Вестник Воронежского государственного университета. Серия: География. Геоэкология, 2020, № 2, с. 35-39. DOI: https://doi.org/10.17308/geo.2020.2/2883

\section{ВВЕДЕНИЕ}

Видимость определяется в качестве такого горизонтального расстояния на котором человек может различить темный объект с достаточным контрастом по отношению к фону [7, 8]. Видимость зависит от оптических свойств компонентов атмосферы, физических свойств наблюдаемого объекта, а также порогового визуального контраста наблюдателя.

Можно указать по меньшей мере два направления исследований зависимости видимости от метеофакторов.

Первое из этих направлений рассматривает задачу исследования зависимости коэффициента мутности атмосферы Ангстрема $\beta$ от количества водяных паров с учетом увлажнения аэрозоля, оп-

(C) Абасзаде Ф.Г., Асадов Х.Г., 2020

E-mail: asadzade@rambler.ru тическая толщина которого определяется по формуле Ангстрема:

$$
\tau_{\alpha}=\beta \cdot \lambda^{-\alpha}
$$

где $\tau_{\alpha}(\lambda)$ - оптическая толщина атмосферного аэрозоля; $\alpha$ - показатель Ангстрема; $\lambda$ - длина волны. Согласно модели Ангстрема $\alpha=1.3$ [5].

Вместе с тем, гигроскопичность многих типов аэрозоля приводит к тому, что такие аэрозоли, поглощая водяные пары, увеличиваются в размерах и тем самым начинают рассеивать еще больше оптической радиации. Наиболее гидроскопичными являются морская соль, углеродные и неорганические аэрозоли.

Согласно модели Louche [4]

$$
\beta=\frac{1}{m_{\alpha} D} \cdot \ln \left(\frac{C}{A-B}\right),
$$


где

$$
\begin{aligned}
& A=\frac{I_{n}}{0.9751 \cdot I_{0} \cdot T_{r} \cdot T_{0} \cdot T_{g} \cdot T_{w}} \\
& B=0.12445 \alpha-0.0162 \\
& C=1.003-0.125 \alpha \\
& D=1.089 \alpha+0.5123
\end{aligned}
$$

где $T_{r}$ - пропускание Релеевского рассеяния;

$T_{0}$ - пропускание атмосферного озона;

$T_{g}$ - пропускание атмосферных газов;

$T_{w}$ - пропускание водяных паров;

$I_{0}$ - прямая радиация.

Согласно первому направлению исследований относительная влажность воздуха является основным фактором, влияющим на видимость, так как из-за гигроскопичности атмосферного аэрозоля под воздействием относительной влажности аэрозольные частицы увеличивают свои размеры, что в конечном счете увеличивает рассеяние ими света и приводит к уменьшению видимости. Согласно $[2,6]$ имеет место следующее соотношение:

$$
\frac{V I S}{V I S_{d}}=0.26+0.4285 \log _{10}(100-R H),
$$

где $V I S$ - видимость; VIS $S_{d}$ - видимость, скорректированная с учетом влияния $R H$.

Вторым направлением исследования зависимости видимости от метеофакторов является исследование изменения видимости под воздействием температуры воздуха. Исследование температурной зависимости отдельных метеорологических показателей позволяет определить температурную зависимость видимости в целом.

Целью настоящей статьи является изучение совместной зависимости метеорологической видимости как от относительной влажности, так и от температуры воздуха.

\section{ИССЛЕДОВАНИЯ ПО ПЕРВОМУ НАПРАВЛЕНИЮ}

Как видно из выражения (7) между видимостью и относительной влажностью имеется обратная зависимость. Проведем дополнительные модельные исследования для подтверждения указанного факта. Согласно [1] (VIS), существует следующая простая зависимость между видимостью и коэффициентом рассеяния аэрозоля $\beta_{s}(R H)$

$$
V I S=\frac{3.91}{\beta_{s}(R H)}
$$

Согласно модели Кларка [1] коэффициент рассеяния аэрозоля является функцией относительной влажности, т. е.

$$
\beta_{s}(R H)=\beta_{s}\left[1-\frac{B}{\ln (R H)}\right]^{\frac{2}{3}},
$$

где $B$ - параметр активации; при $B=0$.

С учетом (8) и (9) получаем

$$
V I S=\frac{3.91}{\beta_{s}\left[1-\frac{B}{\ln (R H)}\right]^{\frac{2}{3}}}
$$

Как видно из выражения (10) увеличение $R H$ приводит к уменьшению видимости. Такой вывод также следует из выражения (7).

\section{ИССЛЕДОВАНИЯ СОВМЕСТНОЙ ЗАВИСИМОСТИ ВИДИМОСТИ ОТ ОТНОСИТЕЛЬНОЙ ВЛАЖНОСТИ И ТЕМПЕРАТУРЫ}

Согласно [3], существует следующая эмпирическая зависимость

$$
R H\left(T_{x}\right)-R H\left(T_{0}\right)=-a_{1}-a_{2}\left(T_{x}-T_{0}\right),
$$

где $R H\left(T_{x}\right)$ - относительная влажность воздуха при температуре воздуха, равной $T_{x} ; R H\left(T_{0}\right)$ - относительная влажность воздуха при температуре воздуха, равной $T_{0}$.

Из (11) получим

$$
R H\left(T_{x}\right)=R H\left(T_{0}\right)-a_{1}-a_{2}\left(T_{x}-T_{0}\right)
$$

Учитывая (12) и (7), получим

$$
\begin{gathered}
\gamma=\frac{V I S}{V I S_{d}}=0.26+0.4285 \log _{10}\left[100-R H\left(T_{0}\right)+\right. \\
\left.+a_{1}+a_{2}\left(T_{x}-T_{0}\right)\right]
\end{gathered}
$$

Рассмотрим вспомогательный показатель $\gamma_{1}=(\gamma-0.26)$.

Допустим осуществляется много шаговые измерения показателя $R H\left(T_{x}\right)$ в интервале $\left(T_{x}-T_{0}\right) \mathrm{c}$ равным шагом $\Delta T$.

При этом количество измерений относительной влажности $R H_{x}$ при каждом шаге $N_{x i}$ пропорционально величине $T_{x}-T_{0}$, т.е.

$$
N_{x i}=\frac{\left(T_{x i}-T_{0}\right)}{\Delta T}
$$

Количество информации, получаемой при измерениях относительной влажности $R H_{x}$ в $i$-м шаге вычислим как

$$
\begin{gathered}
M_{i}=\frac{\left(T_{x i}-T_{0}\right)}{\Delta T}\left[0 . 4 2 8 5 \operatorname { l o g } _ { 1 0 } \left[100-R H\left(T_{0}\right)+\right.\right. \\
\left.\left.+a_{1}+a_{2}\left(T_{x}-T_{0}\right)\right]\right]
\end{gathered}
$$


Введем на рассмотрения функцию

$$
R H\left(T_{0}\right)=f\left(T_{x}-T_{0}\right)
$$

Примем, что функция $f\left(T_{x}-T_{0}\right)$ удовлетворяет следующему условию

$$
\begin{gathered}
\int_{0}^{\left(T_{x}-T_{0}\right) \max } f\left(T_{x}-T_{0}\right) d\left(T_{x}-T_{0}\right)=C ; \\
C=\text { const }
\end{gathered}
$$

Приняв обозначения:

$$
\begin{gathered}
\alpha=T_{x}-T_{0} \\
R H\left(T_{0}\right)=R H_{T_{0}}
\end{gathered}
$$

и далее, интегрируя (15) в интервале $\left(0-\alpha_{\max }\right)$, напишем

$$
\begin{aligned}
F_{1}= & \int_{0}^{\alpha_{\max }} \frac{\alpha}{\Delta T} 0.4285 \log _{10}\left[100-f(\alpha)+a_{1}+\right. \\
& \left.+a_{2}(\alpha)\right] d \alpha+\lambda\left[\int_{0}^{\alpha_{\max }} f(\alpha) d \alpha-C\right],
\end{aligned}
$$

где $\lambda$ - множитель Лагранжа.

Функционал (20) отображает интегральное количество информации, извлекаемое при измерениях относительной влажности с учетом температуры воздуха.

Таким образом, решение задачи оценки информативности температурных приращений видимости с учетом влияния увлажнения сводится к оптимизации целевого функционала (20) в смысле нахождения оптимальной функции $f(\alpha)$, при которой $F_{1}$ достиг бы экстремальной величины. Решение этой задачи с учетом условий уравнения Эйлера - Лагранжа получено в виде:

$$
f(\alpha)=\alpha \cdot C_{1}+C_{2},
$$

где

$$
C_{1}, C_{2}=\text { const }
$$

Можно показать, что при решении (21) целевой функционал (20) достигает максимальной величины.

Следовательно, для достижения максимальной информативности проводимых измерений температуру $T_{x}$ следует выбирать таким образом, чтобы $R H\left(T_{0}\right)$ был пропорционально значению $C_{1}\left(T_{x}-T_{0}\right)$.

\section{ЗАКЛЮЧЕНИЕ}

Показан порядок проведения многошаговых измерений видимости в условиях увеличения температуры воздуха во времени.
Согласно вычисленной закономерности (21) при каждом шаге измерения показателя $\gamma_{1}$, являющеюся смешенной величиной относительной видимости $\frac{V I S}{V I S_{d}}$, величина приращения температуры в следующем шаге должна быть выбрана пропорционально величине относительной влажности в текущем шаге измерений.

\section{СПИСОК ЛИТЕРАТУРЫ}

1. Clark P. A., Harcourt S. A., Macpherson B., Mathison C. T., Cusack S., Naylor M. Prediction of visibility and aerosol within the operational Met Office Unified Model. I: Model formulation and variational assimilation // Q.J. Meteorol. Soc., 2008, vol. 134, pp. 1801-1806. DOI: 10.1002/qj.318. www.interscience.wiley.com

2. Daniel Rosenfeld, Jin Dai, Xing Yu, Zhanyu Yao, Xiajhong Xu, Xing Yang, Chuanli Du. Inverse relations between amounts of air pollution and orographic precipitation // Science, 2007, vol. 315, pp. 396-405.

3. Labajo J.L., F. De Pablo, Garcia E.L. An equation relating temporal changes of relative humidity and temperature: case of variable intervals of time // Atmosfera, 1991, pp. 257-264.

4. Loche A., Maurel M., Simonnot G., Peri G., Iqbal M. Determination of Angstrom turbidity coefficient from direct total solar irradiance measurements // Solar Energy, 1987, vol. 38, pp. 89-96.

5. Lopez G., Battles F. J. Estimate of the atmospheric turbidity from three broad-band solar radiation algorithms. A comparative study // Annales Geophysicae, 2004, vol. 22, pp. 2657-2668.

6. Nishit aman, Kasemsan Manomaiphiboon, Petch Pengchai, Patcharawadee Suwananathada, Jaruwat Srichawana, Nosha Assereh. Long-term Observed visibility in eastern Thailand: Temporal variation, association with air pollutants and meteorological factors and trends // 2019, vol. 122, no. 10. DOI: 10.3390/atmos10030122.

7. Seinfeld J.H., Pandis S. N., John Wiley \& Sons. Atmospheric Chemistry and Physic from Air Pollution to Climate Change, 2nd. New York. NY. USA. 2006. ISBN 9780471720171.

8. Watson J. G. Visibility: Science and regulation // J. Air Waste Manag. Assoc., 2002, vol. 52, pp. 628-713.

Конфликт интересов: Авторы декларируют отсутствие явных и потенциальных конфликтов интересов, связанных с публикацией настоящей статьи.

Поступила в редакиию 19.09.2019 Принята к публикаичи 02.06.2020 


\title{
Issues of Assessing Visibility Taking into Account the Effect of Humidified Aerosol and Air Temperature
}

\author{
F. G. Abaszadeh, H. H. Asadov ${ }^{\circledR}$ \\ National Aerospace Agency, Azerbaijan (1, S. S. Akhundova St., Baku, AZ1115)
}

\begin{abstract}
The article is devoted to the assessment of visibility, taking into account the influence of humidified aerosol and air temperature. It is well known that visibility depends on the optical properties of atmospheric components, the physical properties of the observed object, as well as the threshold visual contrast of the observer. The purpose of this article is to study the joint dependence of meteorological visibility on both relative humidity and air temperature. The scenario of meteorological measurements is considered when multistep measurements of the relative humidity index $R H\left(T_{x}\right)$ are carried out in the temperature range $\left(T_{x}-T_{0}\right)$ with an equal step. It is shown that, in order to achieve maximum information content of the measurements, the temperature $T_{x}$ should be chosen so that $R H\left(T_{0}\right)$ is proportional to the value $\mathrm{C}_{1}\left(T_{x}-T_{0}\right)$. Thus, the procedure for multi-step visibility measurements in conditions of increasing air temperature over time is determined. According to the calculated regularity, at each step of measuring the newly introduced indicator, which is a shifted value of relative visibility, the temperature increment in the next step should be selected proportionally to the value of relative humidity in the current measurement step.
\end{abstract}

Key words: visibility, relative humidity, optimization, aerosol, humidification, measurements.

For citation: Abaszadeh F. G., Asadov H. H. Issues of Assessing Visibility Taking into Account the Effect of Humidified Aerosol and Air Temperature. Vestnik Voronezskogo gosudarstvennogo universiteta. Seria Geografia. Geoekologia, 2020, No. 2, pp. 35-39. (In Russ.) DOI: https://doi.org/10.17308/geo.2020.2/2883

\section{REFERENCES}

1. Clark P. A., Harcourt S. A., Macpherson B., Mathison C. T., Cusack S., Naylor M. Prediction of visibility and aerosol within the operational Met Office Unified Model. I: Model formulation and variational assimilation. $Q . J$. Meteorol. Soc., 2008, vol. 134, pp. 1801-1806. DOI: 10.1002/qj.318. www.interscience.wiley.com

2. Daniel Rosenfeld, Jin Dai, Xing Yu, Zhanyu Yao, Xiajhong Xu, Xing Yang, Chuanli Du. Inverse relations between amounts of air pollution and orographic precipitation. Science, 2007, vol. 315, pp. 396-405.

3. Labajo J.L., F. De Pablo, Garcia E.L. An equation relating temporal changes of relative humidity and temperature: case of variable intervals of time. Atmosfera, 1991, pp. 257-264.

4. Loche A., Maurel M., Simonnot G., Peri G., Iqbal M. Determination of Angstrom turbidity coefficient from direct total solar irradiance measurements. Solar Energy, 1987, vol. 38, pp. 89-96.
5. Lopez G., Battles F. J. Estimate of the atmospheric turbidity from three broad-band solar radiation algorithms. A comparative study. Annales Geophysicae, 2004, vol. 22, pp. 2657-2668.

6. Nishit aman, Kasemsan Manomaiphiboon, Petch Pengchai, Patcharawadee Suwananathada, Jaruwat Srichawana, Nosha Assereh. Long-term Observed visibility in eastern Thailand: Temporal variation, association with air pollutants and meteorological factors and trends. 2019, vol. 122, no. 10. DOI: $10.3390 /$ atmos 10030122 .

7. Seinfeld J. H., Pandis S. N., John Wiley \& Sons. Atmospheric Chemistry and Physic from Air Pollution to Climate Change, 2nd. New York. NY. USA. 2006. ISBN 9780471720171.

8. Watson J.G. Visibility: Science and regulation. $J$. Air Waste Manag. Assoc., 2002, vol. 52, pp. 628-713.

Conflict of interests: The authors declare no information of obvious and potential conflicts of interest related to the publication of this article.

Received: 19.09.2019

Accepted: 02.06.2020

() Abaszadeh F. G., Asadov H.H., 2020

E-mail: asadzade@ rambler.ru

The content is available under Creative Commons Attribution 4.0 License. 
Абасзаде Фуад Габиль оглы

соискатель докторской степени Национального аэрокосмического агентства, г. Баку, Азербайджанская Республика, ORCID: https://orcid.org/0000-0002-4542-6350

Асадов Хикмет Гамидович доктор технических наук, профессор Национального аэрокосмического агентства, г. Баку, Азербайджанская Республика, ORCID: https://orcid.org/0000-0003-1180-1535, e-mail: asadzade@ rambler.ru
Abaszadeh Fuad Gabil oglu

Doctoral student of National Aerospace Agency, Baku, Azerbaijan, ORCID: https://orcid.org/0000-0002-4542-6350

Asadov Hikmat Hamid oglu

Dr. Sci. (Tech.), Professor of National Aerospace Agency, Baku, Azerbaijan, ORCID: https://orcid.org/0000-0003-1180-1535, e-mail: asadzade@ rambler.ru 